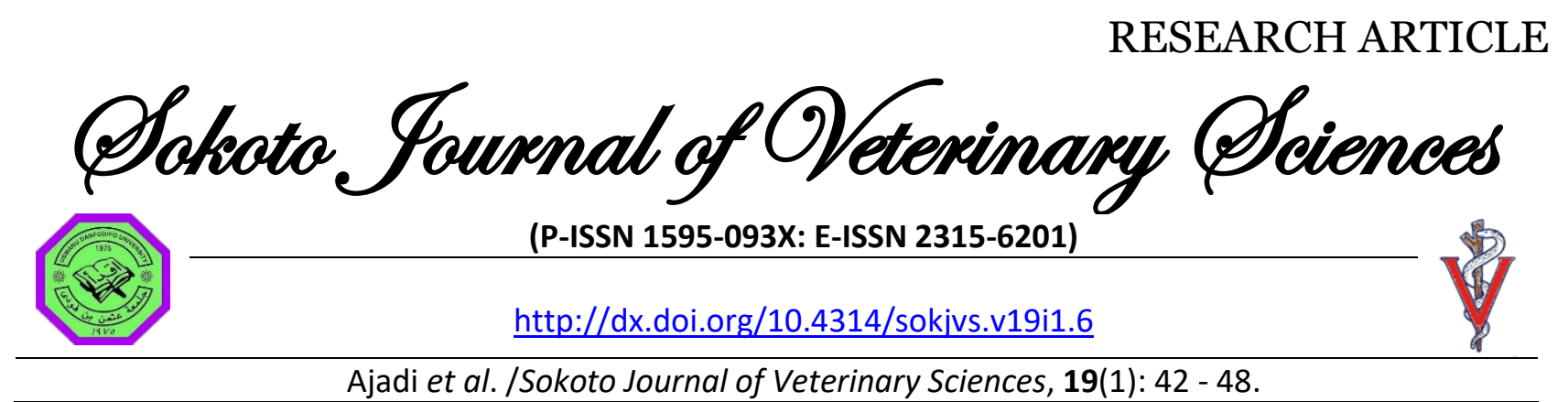

\title{
Phenotypic status of elbow dysplasia in Boerboel dogs in Nigeria
}

\author{
RA Ajadi ${ }^{1 *}$, IO Oyenekan ${ }^{1}$, MM Alabi $^{1}$, OA Makinde ${ }^{1}$, MO Ilugbo $^{2} \&$ SA \\ Koleosho ${ }^{3}$ \\ 1. Department of Veterinary Surgery and Theriogenology, Federal University of Agriculture, Abeokuta, Ogun \\ State, Nigeria \\ 2. Veterinary Teaching Hospital, Federal University of Agriculture, Abeokuta, Ogun State, Nigeria \\ 3. Department of Animal Health, Federal College of Animal Health Production, Moor Plantation, Ibadan, \\ Nigeria
}

*Correspondence: Tel.: + 234 7033800326; E-mail: ade_vsr@hotmail.com

\begin{abstract}
Copyright: (C) 2021 Ajadi et al. This is an open-access article published under the terms of the Creative Commons Attribution License which permits unrestricted use, distribution, and reproduction in any medium, provided the original author and source are credited.

Abstract

Elbow dysplasia (ED) is a developmental, multifactorial, polygenic disease of rapidly growing, large breed of dogs. ED can be a significant health concern for affected dogs because treatments are largely ineffective, making selectively breeding of normal dogs the best option for disease control. Despite the relative availability of data on ED prevalence in other breeds, there is dearth of information on ED prevalence in Boerboel dogs. This study evaluated ED prevalence and relative percentages of different ED grades in Boerboel dogs using radiographs of dogs presented for screening between April 2016 and September 2020. Extended latero-medial, flexed latero-medial and cranio-lateral-caudo-medial oblique radiographs from 64 dogs were reviewed. Radiographs were graded using international elbow working group (IEWG) criteria and grades 0-3 assigned to each joint. ED prevalence expressed as percentages were obtained by dividing the number of dogs with grades 1,2 and 3 by total number of dogs. Age and sex difference in ED prevalence were compared using chi-square test. Differences were considered significant at $P \leq 0.05$. Mean age of the dogs was $2.3 \pm 0.6$ years. Thirty-six (56.3\%) dogs were graded as non-dysplastic, while $28(43.7 \%)$ were graded as dysplastic, with grade $3(46.4 \%)$ accounting for majority of Publication History: ED cases. There were no significant $(p>0.05)$ difference in sex and age prevalence of Received: 25-11-2020 Revised: $10-01-2021$ Accepted: 11-01-2021 ED in the breed. In conclusion, ED prevalence in Boerboel dog is high with majority of dogs having severe grade. Widespread phenotypic screening and strict breeding restrictions are recommended | to control the disease in the breed.
\end{abstract}

Keywords: Boerboel, Dog, Elbow dysplasia, Prevalence, Radiographic screening 


\section{Introduction}

The elbow joint is formed by the articulation of the humeral condyle, the coronoid process of the radius and the anconeal process of the ulna. It is a complex joint comprising of humero-radial, humero-ulnar and radio-ulnar joints (Michelsen, 2013). The joint allows for extension, flexion, pronation and supination. Embryologically, the elbow joint is a diversity of secondary ossification centres in the distal humerus, proximal radius and ulna (Lau et al., 2013). The lateral and medial humeral epicondyle ossifies around sixteen weeks, while the anconeal process fuses by 20 weeks. The fusion of the different ossification centres vary in different breeds and influenced by nutrition and other environmental factors. Disturbance in the process of ossification could result in imbalance in the congruity of the different compartments of the elbow joint resulting in laxity of the elbow joint and chronic osteoarthritis (Lavrijsen et al., 2014).

Elbow dysplasia (ED) is a developmental, multifactorial, polygenic disease of rapidly growing, large breed dogs characterized by joint incongruity, primary lesions (Fragmented Medial Coronoid Process (FMCP), United Anconeal Process (UAP), Osteochondritis (OCD) of humeral epicondyle), and secondary osteoarthritis (Michelsen, 2013). It is the major cause of forelimb lameness in dogs. Although it is majorly a disease of the large breeds such as Rottweiler, Labrador retriever, Bernese Mountain Dog etc., it has been reported in smaller chondrodystrophic breeds such as Daschunds and French bulldog (Narojek et al., 2008). ED is a common endpoint for several genetic disorders of the elbow joint. Three mechanisms have been suggested for the development of elbow dysplasia including osteochondrosis (OC), joint incongruities (Michelsen, 2013) and biomechanical force mismatch across the elbow joint (Lavrijsen et al., 2014). All are hypothesized to occur as a result of a genetic predisposition with secondary environmental influencing factors, such as high energy diets, leading to rapid growth rates or excessive exercise (Baers et al., 2019).

Medial coronoid disease (MCD) is an inherited, multifactorial disorder of canine elbow joints that affects young, large, rapidly growing dog and is a major cause of elbow dysplasia in dogs. The exact aetiology of an MCD is not fully clear, but is thought to be due to lesions of the subchondral bone with abnormal loading leading to primary osseous fissuring, micro crack formation and elbow incongruence (Baers et al., 2019). Fragmented medial coronoid process is the most common cause of elbow dysplasia accounting for about $94 \%$ of positive cases of ED (Narojek et al., 2008).

The anconeal process usually fuses with the proximal metaphysis of the ulna in dogs around 20 weeks of age. If the anconeal process does not fuse with the ulna within 20 weeks, union will not occur spontaneously resulting in a condition called ununited anconeal process (UAP). This condition results in medial to lateral instability of the anconeal process causing irritation, deterioration and secondary degeneration of the articular surfaces of the elbow joint (Michelsen, 2013). UAP is rarely reported in dogs and prevalence has been reported to be $1.5 \%$ (Lavrijsen et al., 2014).

The reported prevalence of ED in UK Labradors was estimated at $17 \%$, while a prevalence of $70 \%$ was reported in Bernese mountain dogs (Woolliams et al., 2011; Lavrijsen et al., 2012; Michelsen, 2013, O'Neill et al., 2020). In a survey of ED among Dutch pure breed dogs, Newfoundland had a $19 \%$ incidence; Golden retrievers had a prevalence of $5.6 \%$, while ED prevalence in Labrador retriever was 5.2\% (Lavrijsen et al., 2014). In a study of pure-bred dogs in South Africa, Rottweiler had the highest incidence of ED at 39\%, while Labrador retriever had a prevalence of 19\% (Kirberger, 2017). In a report based on Orthopaedic Foundation for Animal (OFA) data base, Chowchow (48.6\%) and Rottweiler (38.1\%) had the highest ED prevalence, while Briard (0.2\%) and Cavalier King Charles Spaniel $(0.3 \%)$ had the lowest ED prevalence (Oberbauer et al., 2017).

The Boerboel dog is a rapidly growing big Molloser type of dog reported to be as a result of crossbreed between Bullmastiff and local South African dog breeds like Bullenbijter (Ajadi \& Doyin-Dada, 2019). Even though Boerboels are generally known for their good health, they are reported to suffer from hip and elbow dysplasia (Kirberger \& Stander, 2007). In spite of the susceptibility of Boerboels to ED, there is dearth of information on the prevalence of ED in the breed. In our preliminary study, we reported a prevalence of 25\% for ED in Boerboel dogs (Ajadi \& Doyin-Dada, 2019). In this study, we reviewed the radiographs of the elbow joint of dogs presented for hip and elbow certification between 2016 and 2020 to determine the overall prevalence of $E D$ in the breed, as well as determining the prevalences of UAP and FMCP in the Boerboel dogs. 
Table 1: International Elbow Working Group (IEWG) criteria for elbow grading

\begin{tabular}{|c|c|c|}
\hline IEWG Grade & Description & FCl Criteria \\
\hline 0 & Normal elbow joint & Normal elbow joint, no evidence of incongruency, sclerosis or arthrosis \\
\hline 1 & Mild Arthrosis & $\begin{array}{l}\text { Presence of osteophytes } 2 \mathrm{~mm} \text { high, sclerosis of the base of the } \\
\text { coronoid process - trabecular pattern still }\end{array}$ \\
\hline 2 & $\begin{array}{l}\text { Moderate arthrosis } \\
\text { or Suspected primary } \\
\text { lesion }\end{array}$ & $\begin{array}{l}\text { Visible presence of osteophytes of } 2-5 \mathrm{~mm} \text { high Obvious sclerosis (no } \\
\text { trabecular pattern) of the base of the coronoid processes Step of 3-5 } \\
\mathrm{mm} \text { between radius and ulna (INC). Indirect signs for a primary lesion } \\
\text { (UAP, FCP/MCD, OCD) }\end{array}$ \\
\hline 3 & $\begin{array}{l}\text { Severe arthrosis or } \\
\text { evident primary } \\
\text { lesion }\end{array}$ & $\begin{array}{l}\text { Presence of osteophytes of }>5 \mathrm{~mm} \text { high Step of }>5 \mathrm{~mm} \text { between radius } \\
\text { and ulna (obvious INC) Obvious presence of a primary lesion (UAP, FCP, } \\
\text { OCD) }\end{array}$ \\
\hline
\end{tabular}

\section{Materials and Methods \\ Animals}

Extended latero-medial, flexed lateromedial and cranio-lateral-caudo-medial oblique radiographs from Boerboel dogs presented for hip and elbow certification between April 2016 and September 2020 were reviewed. The radiographs were obtained following premedication with intramuscular injections of $0.5 \mathrm{mg} / \mathrm{kg}$ of $2 \%$ xylazine hydrochloride (Xylazine 20 Inj ${ }^{\circledR}$, Kepro, Holland) and $0.04 \mathrm{mg} / \mathrm{kg}$ atropine sulphate $\left(\right.$ Atocan $^{\circledR}$, Sishui Xierkang Pharma, China) and anaesthesia with intravenous injection of $4.0 \mathrm{mg} / \mathrm{kg}$ of $1 \%$ propofol (Diprivan, ICl - Zeneca Pharmaceuticals). Once the dog was anaesthetized, they were positioned on the radiographic table in right or left lateral recumbency with the lowermost limb being the one under examination. The elbows were placed directly on the cassette without the use of grid and the beam collimated and centred on the medial humeral condyle. For the cranio-lateral-caudomedial view, the elbow was pronated inward at an angle of 45 degrees. All the radiographs were obtained using a digital X-ray machine (Brivio XR 115, GE Health Care Services, General Electric Company, India). Exposure factors ranged from 10$16 \mathrm{mAs}$ and 55-65 KvP, depending on the size of the dog. Informed consent was obtained for the clientowned animals included in this study.

\section{Elbow dysplasia grading}

The radiographs were graded using the international elbow working group (IEWG) criteria (Table 1). Each joint was assigned to one of the four grades $(0-3)$. Where the grading for the two joints varied, the worst of the grade assigned to either of the limb was recorded as the final grade for the dog. Elbow grade 0 was considered as normal joints (non-dysplastic), while grades 1 - 3 represent mild, moderate and severe dysplasia, respectively. All the radiographs were graded by a single scrutineer at the same time to remove inter and intra- observer variability.

\section{Data analysis}

ED prevalence expressed as percentages was obtained by dividing the number of dogs that had grades 1,2 and 3 by the total number of dogs. Age and sex difference in the prevalence of ED were compared using chi-square test. The prevalence of primary lesion (UAP and FMCP) was calculated. In addition, relative percentages were calculated for ED grades 1, 2, and 3. Differences were considered significant at $P<0.05$. Statistical analyses were performed using Graph-Pad Prism 6 Software, La Jolla, USA, CA).

\section{Results}

Radiographs from a total of sixty-four (64) dogs were reviewed. This was made up of $27(42.2 \%)$ male and 37 (57.8\%) female Boerboel dogs. The mean \pm standard deviation age of the dogs was $2.3 \pm 0.6$ years with age ranging between 1 and 6 years. Of all the radiographs reviewed, 36 (56.3\%) were graded 


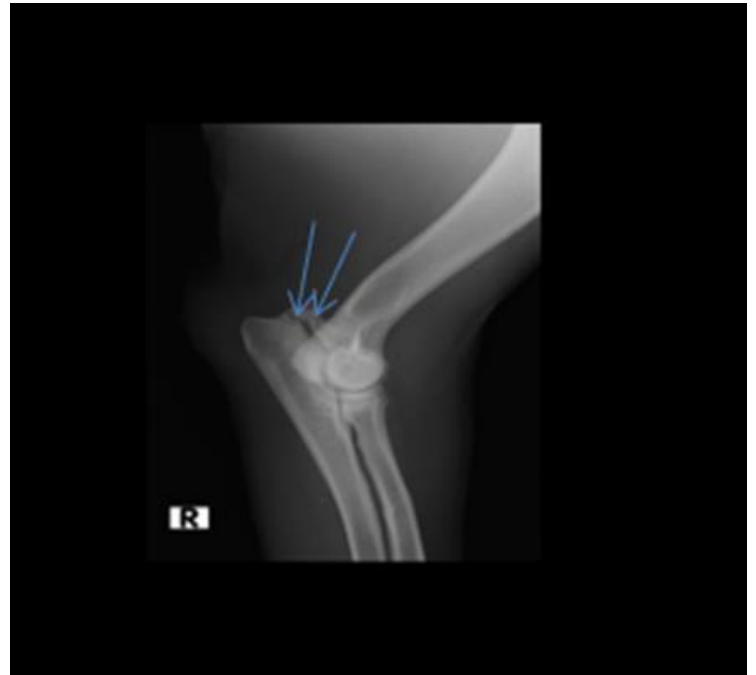

Plate I: Extended latero-medial radiograph of Boerboel dog showing un-united anconeal process (arrows)

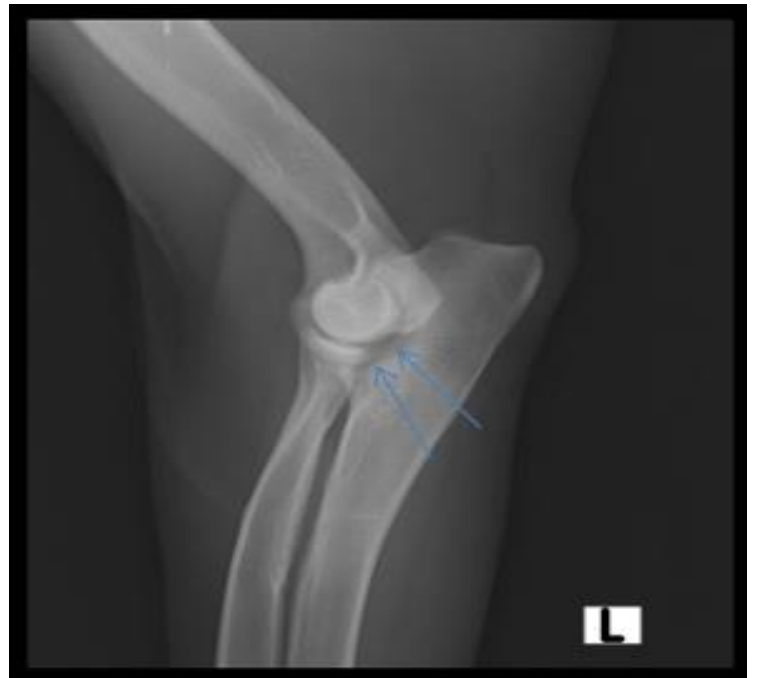

Plate II: Extended latero-medial radiograph of Boerboel dog showing fragmented medial coronoid process (arrows)

Table 4: Relative percentage of primary lesions of elbow dysplasia in Boerboel dogs

\begin{tabular}{lll}
\hline Grades & Number & Relative Percentage (\%) \\
\hline OCD & 0 & 0 \\
UAP & 2 & 15.4 \\
FMCP & 11 & 84.6 \\
\hline OCD: Osteochondritis dissecans; UAP: Ununited Anconeal Process; FMCP: Fragmented Medial Coronoid Process
\end{tabular}

as non-dysplastic, while 28(43.7\%) were graded as dysplastic. Table 2 and 3 showed the prevalence of the different elbow grades in the Boerboel dogs. Dogs with grade 1 (25.0\%) accounted for the least of the ED cases, while dogs with grade 3 (46.4\%) accounted for most of the ED cases. FMCP (Plate I) accounted for most of the primary lesions, while UAP (Plate II) had a relative percentage of $15.4 \%$ (Table 4 ).

There were no record of dogs with radiographic evidence of OCD in the study. The sex prevalence of ED in Boerboel dogs is shown in Table 5. Prevalence of ED did not differ significantly $(p>0.05)$ between female and male Boerboel dogs. Similarly, there was no significant $(p>0.05)$ difference in the prevalence of ED between dogs that are $1-2$ years of age and dogs that are $3-6$ years of age (Table 6 ). Bilateral cases of ED were higher than unilateral cases in the Boerboel dogs (Table 7).

\section{Discussion}

According to the IEWG regulations, official ED screening requires at least two but preferably four radiographic views of each of the elbows. The
Table 5: Sex prevalence of hip dysplasia in Boerboel dogs

\begin{tabular}{llll}
\hline Sex & Number & Dysplastic & Prevalence $(\%)$ \\
\hline Male & 14 & 13 & 20.3 \\
Female & 22 & 15 & 23.4 \\
Total & 36 & 28 & 43.7 \\
\hline
\end{tabular}

$P$ value $=0.568$

Table 6: Age prevalence of elbow dysplasia in Boerboel dogs

\begin{tabular}{llll}
\hline Age (Years) & Normal & Dysplastic & Prevalence $(\%)$ \\
\hline $1-2$ & 22 & 18 & 28.1 \\
$3-6$ & 14 & 10 & 15.6 \\
Total & 36 & 28 & 43.7 \\
\hline
\end{tabular}

$P$ value $\equiv 0.318$ recommended views include extended lateromedial, flexed latero-medial, cranio-caudal and craniolateral-caudomedial oblique views. The number of views used for elbow screening varies from one country to another and between breeds. In Netherlands, four radiographic views are required for scoring in Labrador retriever, Golden retriever, Bernese Mountain dogs, Rottweilers, German Shepherd dogs and Bordeaux Dogs, while other breeds only require extended latero-medial and craniolateral-caudomedial views (Lavrijsen et al., 
Table 7: Relative percentage of bilateral versus unilateral cases of elbow dysplasia in Boerboel dogs

\begin{tabular}{lll}
\hline Grades & Number & Relative Percentage (\%) \\
\hline Unilateral & 13 & 46.4 \\
Bilateral & 15 & 53.6 \\
\hline
\end{tabular}

2014). In South Africa, extended latero-medial and craniolateral-caudomedial oblique views are used for elbow screening (Kirberger, 2017). In this study, three radiographic views namely extended lateromedial, flexed latero-medial and craniolateralcaudomedial oblique views were used. The use of these three radiographic views enables us to be able to recognize primary lesions better compared to when only the flexed latero-medial view was used (Ajadi \& Doyin-Dada, 2019). It is of noteworthy that in spite of these three radiographic views, we were unable to recognize any dog with OCD. Use of endoscopy and computed tomography has been reported to be more sensitive in the diagnosis of elbow disorders (Meyer-Lindenberg et al., 2002). The prevalence of ED for Boerboel dogs in this study was higher when compared to several other breeds such as Labrador retriever (5.2\%), Golden retriever (5.6\%), Bernese mountain dog (13.9\%) and Estrela Mountain dogs (16.5\%) (Lavrijsen et al., 2012; Alves-Pimenta et al., 2013), but similar to that reported for Rottweiler (39\%) and chowchow (47.2) (Kirberger \& Stander, 2007; Lavrijsen et al., 2012; Baers et al., 2019). The ED prevalence for Boerboel dogs in this study is higher when compared to our previous study (Ajadi \& Doyin-Dada, 2019), but similar to that reported for Boerboel dogs in South Africa (Kirberger \& Stander, 2007). The high ED prevalence in this study might be associated to the additional radiographic projections which allowed for recognition of dogs with primary lesion unlike, the previous study in which only flexed latero-medial view was used. It is noteworthy that ED prevalence for a breed varies from one country to another depending on the level of breeding selection put in place. For instance, ED prevalence in Labrador retriever (9.1\%) from the USA (Baers et al., 2019) was lower than that reported for the same breed in South Africa (Kirberger, 2017).

Dogs with grade 3 (Severe) ED accounted for most of the cases of ED in this study. This finding is contrary to that reported in Estrela mountain dogs in which dogs with grade 1 (Mild) ED accounted for the majority of the cases of ED in the breed (AlvesPimenta et al., 2013), but similar to that reported for Boerboel dogs from South Africa (Kirberger \& Stander, 2007). Several factors such as absence of breeding restrictions, nutrition and type of housing might have contributed to the high prevalence of severe ED form in the Boerboel dogs. It is worthy of note that phenotypic screening for HD and ED started in Boerboel dogs in Nigeria in 2016, while very few breeders who wanted to have their dogs registered with the Kennel Union of South Africa (KUSA) have embraced the screening. The high percentage of dogs with severe grade of ED thus implied that strict breeding restriction using phenotypic ED screening should be put in place to reduce the prevalence of ED in the breed.

In several reports, FMCP is the most reported primary lesions associated with ED in dogs (Lavrijsen et al., 2014; O'Neil et al., 2020). Similarly, in this study FMCP accounted for $84.6 \%$ of the primary cause of ED in the Boerboel dogs. On the contrary, in this study, the relative percentage of cases of UAP was higher in the Boerboel dogs than that reported for other breeds. Breed disposition for UAP have been reported in German shepherd dog due to broad chondral junction in association with accelerated pattern of skeletal maturation (Janutta \& Distl, 2006). No case of OCD was recorded in this study and may be associated with the relatively young age of the dog at radiography and the fact that majority of the dogs do not have history of lameness at the time of radiography. It may also be that it was difficult to recognize $O C D$ in the dogs because of the projections used (Janutta \& Distl, 2006).

The effect of sex on the prevalence of ED varies depending on the breed of the dog. In Labrador retrievers in the US, significant sex predisposition for ED has been reported in males (Oberbauer et al., 2017). This was not observed in Golden retrievers, Newfoundland and Bernese mountain dog. In a South African study of ED, male Rottweilers had a higher ED prevalence than the female but there was no significant difference in ED prevalence in Labrador retrievers. Similarly, Rottweiler males had significantly higher ED numerical scores than females (Kirberger, 2017). In this study, there was no significant difference in the prevalence of ED between male and female Boerboel dogs. This finding is also similar to that reported in our previous study (Ajadi \& Doyin-Dada, 2019).

Occurrence of ED in dogs is biphasic. Most cases first present at 6-12 months of age, while some dogs present later in life ( $>6$ years old), with clinical 
manifestations of medial coronoid disease and little or no prior history of lameness (Lavrijsen et al., 2014). Age was reported to have minor impact on elbow ratings in 16 different breeds with Bichon, Chinese shar-pei, and Akita, reported to have more favorable elbow ratings with increasing age. There is no significant difference in the prevalence of ED between Boerboel dogs (1-2 years) and those 3-6 years of age (Kirberger, 2017). Majority of the dogs screened in this study were between 1 and 3 years of age. Older dogs were not presented for screening probably because the breeders might have culled such dogs from breeding at that age. It will be important to determine the prevalence of ED in Boerboel dogs that are older than 6 years

Manifestation of ED can be either unilateral or bilateral, with bilateral occurrence being more common (Rezende et al., 2012). In this study, bilateral cases of ED accounted for 53.6 percent of the cases of ED in the Boerboel dogs. This finding is similar to that reported for Estrela mountain dogs (Alves-Pimenta et al., 2013). It was suggested that bilaterality of ED can increase the overall risk of ED in the offspring if one or both parents are affected (Baers et al., 2019). In some countries mating between dogs with grade 1 ED is allowed. This will imply that there is increased risk of the offspring having ED if one or both parents with grade 1 ED is bilateral than when they are unilateral. Thus, understanding the impact of the overall heritability of both bilateral and unilateral elbow dysplasia is important in guiding breeding decisions in order to reduce the incidence of the disease in future generations of dogs.

Finally, there were a number of limitations to the study thus requiring that the result be interpreted with cautions. The number of radiographs reviewed is relatively small compared to the number of Boerboel dogs used for breeding in Nigeria. The relatively small sample size might have accounted for the zero-prevalence rate recorded for OCD. However, it should be noted that phenotypic screening for HD and ED is relatively new in Nigeria and there is no breeding regulation that makes HD and ED screening compulsory for breeding. Another limitation to the study was that the review covered only a short period of time. This also contributed to the relatively small sample size of the study. In addition, majority of the dogs radiographed were young and only few numbers of older dogs were presented for radiography. Finally, only one scrutineer graded all the radiographs and this might have influenced the grades allotted to each dog.
However, in spite of these limitations the result of this study is still consistent with previous prevalence report of ED in Boerboel dogs from South Africa (Kirberger \& Stander, 2007).

In conclusion, the result of this study showed that the prevalence of ED in Boerboel dog was high with greater percentage of the dog having severe grade. ED can be a significant health concern for the affected dogs and treatments are largely ineffective; thus, controlling this disease through selectively breeding of normal dogs using results of phenotypic screening appears to be the best option available to breeders. Thus, the control of the disease falls to the breeder's responsibility to have dogs radiographed and make appropriate decisions on the type of sire and dam to be used for breeding. It is recommended that widespread phenotypic screening, and strict breeding restrictions be introduced in the breed in order to reduce the prevalence of ED in the breed.

\section{Conflicts of Interest}

The authors declare no conflict of interest.

\section{References}

Ajadi RA \& Doyin-Dada OA (2019). Preliminary Evaluation of prevalence of hip and elbow dysplasia in Boerboel dogs. Sokoto Journal of Veterinary Sciences, 17(2): $45-53$.

Alves-Pimenta SB, Colaco AM \& Ginja MM (2013). Prevalence and Breeding values of elbow dysplasia in Estrela mountain dogs. Veterinarni Medicina, 58(9): 484-490.

Baers G, Keller GG, Famula TR \& Oberbauer AM (2019). Heritability of unilateral elbow dysplasia in the dog: A retrospective study of sire and dam influence. Frontiers in Veterinary Sciences, 6(422): 1 - 10.

Janutta V \& Distl O (2006). Review on canine elbow dysplasia: pathogenesis, diagnosis, prevalence and genetic aspects. Deutsche Tierarztliche Wochenschrift, 115(5): 172 181.

Kirberger RM \& Stander N (2007). Incidence of canine elbow dysplasia in South Africa. Journal of the South African Veterinary Association, 78(2): 59-62.

Kirberger RM (2017). Phenotypic hip and elbow dysplasia trends in Rottweilers and Labrador retrievers in South Africa (20072015): Are we making progress? Journal of the South African Veterinary Association, 88(0): e1-e10 
Lau SF, Hazewinkel HAW, Grinwis GCM, Wolschrijn CF, Siebelt M, Vernooij JCM, Voorhout G \& Tryfonidou MA (2013). Delayed endochondral ossification in early medial coronoid disease (MCD): A morphological and immunohistochemical evaluation in growing Labrador retrievers. Veterinary Journal, 197(3): 731-738.

Lavrijsen IC, Heuvena HC, Voorhout G, Meij BP, Theyse LF, Leegwater PA \& Hazewinkel HA $W$ (2012). Phenotypic and genetic evaluation of elbow dysplasia in Dutch Labrador Retrievers, Golden Retrievers, and Bernese Mountain dogs. Veterinary Journal, 193(2):486 - 92.

Lavrijsen ICM, Heuvena HCM, Meija BP, Theysea LFH, Nap RC, Leegwatera PAJ \& Hazewinkel HAW (2014). Prevalence and co-occurrence of hip dysplasia and elbow dysplasia in Dutch pure-bred dogs. Preventive Veterinary Medicine, 114(2014): 114-122

Meyer-Lindenberg A, Langhann A, Fehr M \& Nolte I (2002). Prevalence of fragmented medial coronoid process of the ulna in lame adult dogs. Veterinary Record, 151(8): 230-234.
Michelsen J (2013). Canine elbow dysplasia: Aetiopathogenesis and current treatment recommendations. The Veterinary Journal, 196(1):12 - 19

Narojek T, Fiszdon K \& Hanysz E (2008). Canine elbow dysplasia in different breeds. Bulletin of the Veterinary Institute in Pulawy, 52(1): $169-173$.

Oberbauer AM, Keller GG \& Famula TR (2017). Longterm genetic selection reduced prevalence of hip and elbow dysplasia in 60 dog breeds. PLOS ONE, 12 e0172918;

O'Neill DG, Brodbelt DC, Hodge R, Church BD \& Meeson RL (2020). Epidemiology and clinical management of elbow joint disease in dogs under primary veterinary care in the UK. Canine Medicine \& Genetics, 71(2020): 1 - 15.

Rezende CMF, Melo EG, Malm V \& Gheller VA (2012). Arthroscopical treatment of elbow joint disease. Arquivo Brasileiro de Medicina Veterinaria e Zootecnia, 64(1): $9-14$.

Woolliams JA, Lewis TW \& Blott SC (2011). Canine hip and elbow dysplasia in UK Labrador retrievers. Veterinary Journal, 189(2): 16917. 